\title{
Nul-Go: Recursive Non-Local Encoder-Decoder Network for Retinal Image Non-Uniform Illumination Removal
}

\author{
Chongyi Li \\ Nanyang Technological University \\ lichongyi25@gmail.com
}

\author{
Huazhu Fu* \\ Inception Institute of Artificial \\ Intelligence (IIAI) \\ hzfu@ieee.org
}

\author{
Runmin Cong* \\ Institute of Information Science, \\ Beijing Jiaotong University \\ rmcong@bjtu.edu.cn
}

\author{
Zechao Li \\ Nanjing University of Science and \\ Technology \\ zechao.li@njust.edu.cn
}

\author{
Qianqian Xu \\ Institute of Computing Technology, \\ Chinese Academy of Sciences \\ xuqianqian@ict.ac.cn
}

\begin{abstract}
Retinal images have been widely used by clinicians for early diagnosis of ocular diseases. However, the quality of retinal images is often clinically unsatisfactory due to eye lesions and imperfect imaging process. One of the most challenging quality degradation issues in retinal images is non-uniform which hinders the pathological information and further impairs the diagnosis of ophthalmologists and computer-aided analysis. To address this issue, we propose a nonuniform illumination removal network for retinal image, called NuIGo, which consists of three Recursive Non-local Encoder-Decoder Residual Blocks (NEDRBs) for enhancing the degraded retinal images in a progressive manner. Each NEDRB contains a feature encoder module that captures the hierarchical feature representations, a non-local context module that models the context information, and a feature decoder module that recovers the details and spatial dimension. Additionally, the symmetric skip-connections between the encoder module and the decoder module provide long-range information compensation and reuse. Extensive experiments demonstrate that the proposed method can effectively remove the nonuniform illumination on retinal images while well preserving the image details and color. We further demonstrate the advantages of the proposed method for improving the accuracy of retinal vessel segmentation. https://li-chongyi.github.io/Proj_ACMMM20_NuI-Go
\end{abstract}

\section{CCS CONCEPTS}

\section{- Computing methodologies $\rightarrow$ Computer vision problems.}

\section{KEYWORDS}

retinal image enhancement; non-uniform illumination removal; deep learning

\footnotetext{
${ }^{*}$ Corresponding author
}

Permission to make digital or hard copies of all or part of this work for personal or classroom use is granted without fee provided that copies are not made or distributed for profit or commercial advantage and that copies bear this notice and the full citation on the first page. Copyrights for components of this work owned by others than ACM must be honored. Abstracting with credit is permitted. To copy otherwise, or republish, to post on servers or to redistribute to lists, requires prior specific permission and/or a fee. Request permissions from permissions@acm.org.

MM '20, October 12-16, 2020, Seattle, WA, USA

(C) 2020 Association for Computing Machinery.

ACM ISBN 978-1-4503-7988-5/20/10 .. \$15.00

https://doi.org/10.1145/3394171.3413928
ACM Reference Format:

Chongyi Li, Huazhu Fu, Runmin Cong, Zechao Li, and Qianqian Xu. 2020. NuI-Go: Recursive Non-Local Encoder-Decoder Network for Retinal Image Non-Uniform Illumination Removal. In Proceedings of the 28th ACM International Conference on Multimedia (MM '20), October 12-16, 2020, Seattle, WA, USA. ACM, New York, NY, USA, 9 pages. https://doi.org/10.1145/3394171. 3413928

\section{INTRODUCTION}

Retinal images play important roles in diagnosing and monitoring retinal diseases in early stages, including glaucoma, diabetic retinopathy, pathological myopia, etc. $[1,5,30]$. However, retinal images often suffer from the issues of non-uniform illumination, low illumination, low contrast, and detail blurring due to imperfect imaging process $[15,45,51]$. Such quality degraded retinal images significantly impair the diagnosis of ophthalmologists and also affect the performance of computer-aided analysis of retinal diseases [4], such as retinal vessel segmentation, optical disc segmentation, and vascular structure recognition. One of the most challenging quality degradation issues in retinal images is the non-uniform illumination which is the main focus in this paper. It is necessary to remove the non-uniform illumination on retinal images before using them in diagnosis.

Due to the limited generalization capability, the traditional image enhancement methods (e.g., Histogram Equalization (HE), Contrast Limited Adaptive Histogram Equalization (CLAHE), and Gamma correction) that either adjust the histogram distribution of images or globally correct the pixel values cannot be directly extended to retinal images. Sometimes, these traditional methods even tend to introduce unfriendly artifacts when they are used to process the low-quality retinal images. These artifacts severely affect the accuracy of diagnosis, which cannot be used in practical conditions. To this end, several image enhancement methods specially designed for retinal images have been proposed [4, 8, 40, 51]. Unfortunately, the performance of retinal image enhancement remains largely unsatisfactory, especially in practical applications, such as vessel segmentation, vascular structure recognition, and disease diagnosis. In addition, recent years have witnessed the significant success of deep learning in medical image processing [9, 19, 36, 41, 50]. However, deep learning-based retinal image enhancement receives less attention due to lacking sufficient training data and effective 


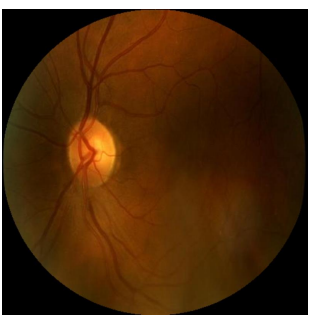

(a) Retinal image

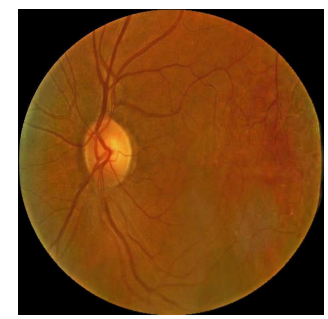

(b) NuI-Go
Figure 1: Sample result on a real retinal image. The NuI-Go can correct the illumination of retinal image while well preserving its details and color.

network architectures. In this paper, we will address these issues and provide the first deep learning-based solution for non-uniform illumination removal in retinal images.

Different from existing methods, our method combines the capacity of neural networks with image physical formation model to study the issue of retinal image non-uniform illumination, which leads to more reasonable and reliable enhancement performance. Concretely, we first approximate the retinal image formation model with non-uniform illumination by combining the domain knowledge of retinal images with the human-lens attenuation and scattering model. With the synthetic retinal images with degradations, a non-local encoder-decoder recursive network architecture is proposed to learn the mapping between the retinal image with nonuniform illumination and the corresponding clear counterpart. The degraded retinal image is enhanced by recursive Non-local EncoderDecoder Residual Blocks (NEDRBs) in a progressive manner. Extensive experiments demonstrate that the proposed method achieves the state-of-the-art performance on both real and synthetic images in qualitative and quantitative metrics. In addition, we further demonstrate the advantages of the proposed method for subsequent retinal vessel segmentation task. In Figure 1, we present a sample result of our NuI-Go. Our NuI-Go can effectively correct the illumination of the retinal image while well preserving its detail and color information.

The contributions of this work are as follows.

- This work is the first attempt to provide a deep learning solution for the problem of retinal image non-uniform illumination removal, which can effectively correct the illumination of a retinal image while well preserving the original details and color appearances.

- We propose a simple yet effective non-local encoder-decoder network to progressively enhance degraded retinal images, which integrates local and non-local information simultaneously.

- Benefiting from novel network architecture and physical model-based non-uniform illumination synthesis strategy, our method achieves impressive performance on retinal image non-uniform illumination removal.

\section{RELATED WORK}

In this paper, we mainly focus on image light enhancement methods that can be categorized into traditional image enhancement methods and retinal image enhancement methods.

Traditional Image Enhancement Methods. HE-based methods [2, $6,18,22,26,27,44]$ adjust the histogram distribution of image to improve its contrast. However, HE-based methods tend to overenhance the images due to impertinently changing the dynamic range of image and some local HE methods are time-consuming. Unlike the HE-based methods, the Retinex theory [23] assumes that the image can be decomposed into reflectance and illumination, and has been widely used in low-light image enhancement thanks to its physical interpretability. Generally, the reflectance component is treated as the enhanced image [10, 14, 31, 32, 39, 47]. However, there are some limitations of Retinex-based image enhancement due to the gaps between real-world high-quality images and the reflectance component. Thus, the results of Retinex-based methods look unnatural and unrealistic. In addition, the last decade has witnessed an increasing interest in image light enhancement $[3,13,24,29,33,38,46]$, especially with the emergence of deep learning.

Retinal Image Enhancement Methods. Recently, some methods specially designed for retinal image enhancement have been proposed. Cheng et al. [4] proposed a structure-preserving guided retinal image filter, called SGRIF. Feng et al. [8] used the Coutourlet transform to achieve retinal image contrast enhancement. In [34], a non-uniform illuminated fundus image enhancement method was proposed, which reduces the blurriness of fundus images based on the cataract physical model and enhances the images with an objective on contrast perfection with no preamble of artifacts. Saha et al. [40] proposed a two-step method to process the non-uniform or poor illumination of retinal images, including an illumination correction algorithm and a color restoration algorithm. Zhou et al. [51] designed a retinal image enhancement method based on luminosity and contrast adjustment.

Despite the prolific work, the performance of retinal image enhancement is still far from satisfactory and practical. With the rise of deep learning technology [7, 12, 16, 25, 28], we see a new dawn in performance improvement; however, deep learning-based retinal image enhancement has not been fully explored. To the best of our knowledge, the proposed NuI-Go network is the first deep learningbased non-uniform illumination removal method for retinal images. Besides, the proposed retinal image non-uniform illumination synthesis strategy can be used for deep models training and also for the evaluations of retinal image enhancement methods.

\section{PROPOSED METHOD}

The network architecture is presented in Figure 2. As illustrated, the NuI-Go network consists of three recursive non-local encoderdecoder residual blocks (NEDRBs) that share the same structure. It takes a degraded retinal image as input and produces a set of enhanced results in a coarse-to-fine scheme. The last output is treated as our final enhanced result. In what follows, we explain the NuI-Go network structure, the loss functions, and the non-uniform illumination retinal image synthesis approach in detail. 


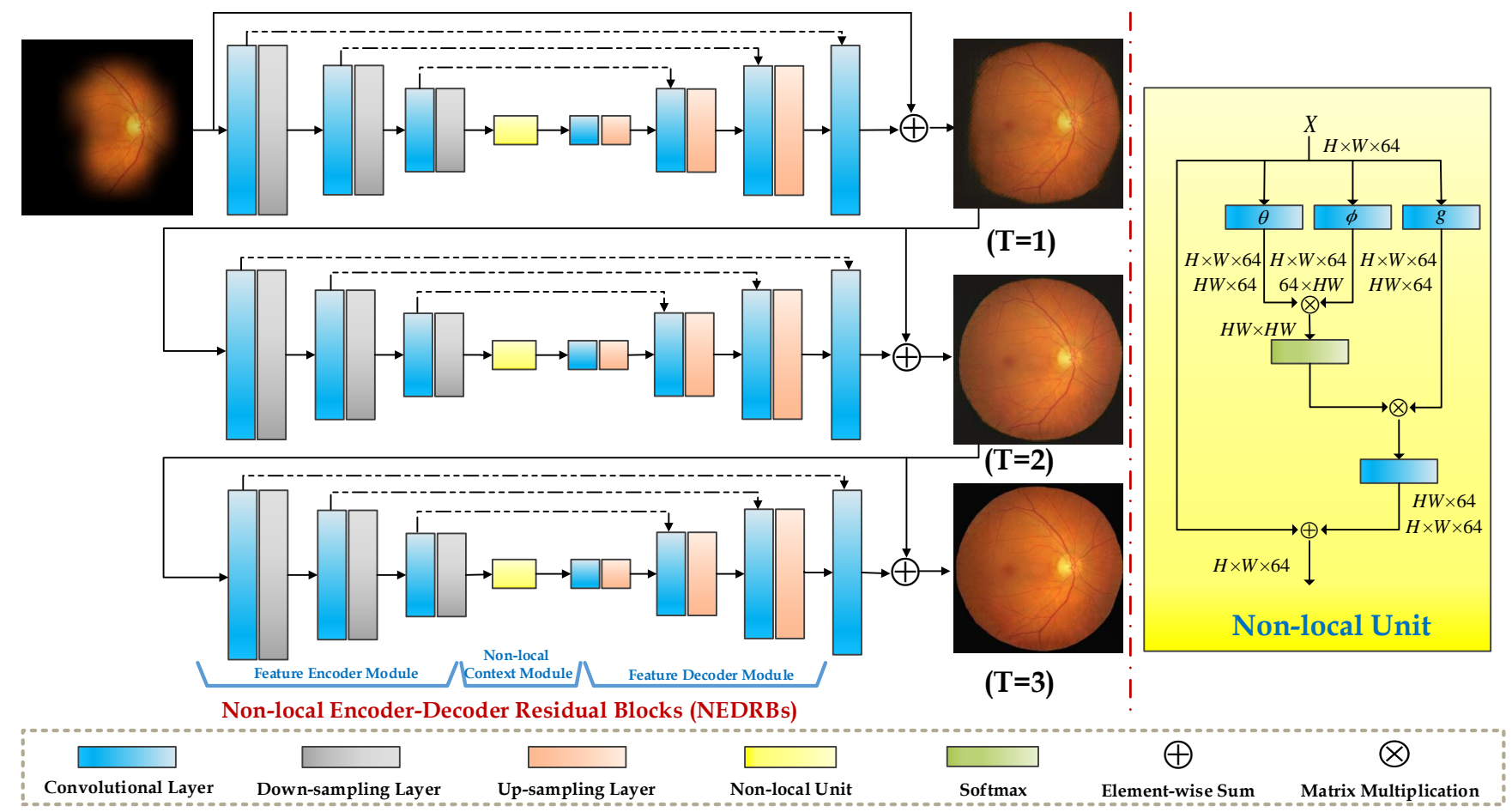

Figure 2: The network architecture of NuI-Go. Left side: three recursive NEDRBs, where each NEDRB contains a feature encoder module, a non-local context module, and a feature decoder module. Right side: the details of non-local unit, where “ $H \times W \times 64$ " denotes height $\times$ width $\times 64$ channels of feature maps $X$ (reshaping is performed when noted).

\subsection{Proposed NuI-Go Network}

We suggest tackling the retinal image non-uniform illumination problem in multiple stages, where a simple yet effective recursive non-local encoder-decoder residual blocks (NEDRB) is used at each stage. Such a recursive manner has several advantages than deeper and more complex networks:

(1) it is computationally efficient;

(2) it is easy to be trained; and

(3) it allows to stop the inference at any stage in practical applications (i.e., our method provides a trade-off between inference time and accuracy).

Note that, the performance gains by adding more stages $(\mathrm{T}>3)$ will become marginal, but at the cost of more training and inference time. Thus, we choose the third stage's result as our final result. The stage-wise results are provided in Figure 3. As shown, the retinal images with non-uniform illumination are gradually becoming clear after stage-by-stage enhancement. Next, we will explain the proposed NEDRB.

NEDRB enjoys an encoder-decoder structure with an embedded non-local unit, which contains a feature encoder module that captures the hierarchical feature representations, a non-local context module that models the context information, and a feature decoder module that recovers the details and spatial dimension. More specifically, the input image is first fed to the feature encoder module that is built by three convolutional layers followed by $2 \times$ down-sampling operation:

$$
F_{\text {enc }}=g\left(f_{3}\left(g\left(f_{2}\left(g\left(f_{1}(I)\right)_{\downarrow}\right)\right)_{\downarrow}\right)\right)_{\downarrow},
$$

where $f$ and $g$ are the notation for the convolution and ReLU actication function, respectivaly, $I$ is the input image, $\downarrow$ denotes the $2 \times$ down-sampling operation. After that, the encoder features $F_{e n c}$ is forwarded to the non-local context module, which can be expressed as:

$$
F_{n l u}=N L U\left(F_{\text {enc }}\right) \text {, }
$$

where $N L U$ represents the operations in the non-local unit. The retinal image shares structured texture and similar color, which provides a strong prior for correcting the non-uniform illumination regions by the context. Therefore, instead of employing the multi-scale convolution to extract local context information used in traditional encoder-decoder networks, we incorporate the nonlocal unit [48] into the proposed encoder-decoder structure, which enlarges the receptive field to the entire image. Our intuition is to incorporate non-local features into local encoder-decoder features for building richer feature representations that combine both non-local and local information. As shown in Figure 2(right), the non-local unit computes the response at a position as weighted sum of the features at all spatial positions.

Specifically, it can be implemented by simple convolution, softmax, etc. (for embedded Gaussian version's non-local unit), where the unknown parameters can be adaptively learned. Embracing the 

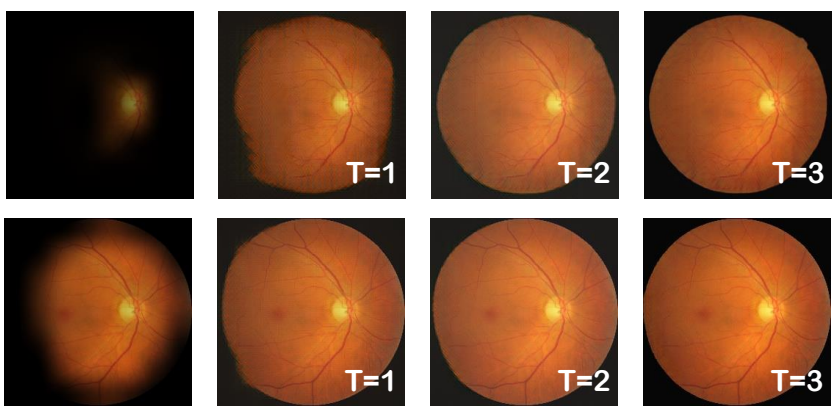

Figure 3: Stage-wise results of the NuI-Go network. From left to right are the input retinal images, the results of stage $T=1$, 2 , and 3 , respectively.

benefit of non-local context information which captures the longrange feature correlation, our network can effectively focus on the non-uniform illumination regions and predict the potential color and details by the long-range dependencies (context modeling).

At last, the features $F_{n l u}$ are passed through decoder module. Coupled with the symmetric skip connections, the operations in decoder module can be expressed as:

$$
\begin{aligned}
& R=I+ f_{7}\left(\left\{f _ { 6 } \left(\left\{g \left(f _ { 5 } \left(\left\{g\left(f_{4}\left(F_{n l u}\right)\right)_{\uparrow}\right.\right.\right.\right.\right.\right.\right. \\
&\left.\left.\left.\left.\left.\left.\left.\left., g\left(f_{3}\right)\right\}\right)\right)_{\uparrow}, g\left(f_{2}\right\}\right)\right)\right)_{\uparrow}, g\left(f_{1}\right)\right\}\right),
\end{aligned}
$$

where $R$ represents the reconstructed result, $\dot{+}$ denotes the elementwise addition, $\uparrow$ is the $2 \times$ up-sampling operation, and $\{$,$\} stands$ for the concatenation operation along the channel dimention. In our recursive structure, we repeat the non-local encoder-decoder residual block three times.

In summary, the encoder-decoder structure can fully exploit hierarchical feature representations and reconstruct the enhanced results while the non-local unit captures long-range dependencies for spatial context modeling. Additionally, the symmetric skipconnections provide long-range information compensation and reuse. Finally, we enforce the NEDRB to learn the difference between the degraded retinal image and its ground truth (residual learning [16]), which facilitates gradient back-propagation and pixel-wise correction.

In the NuI-Go, the down-sampling layer is implemented by $2 \times$ max pooling while the up-sampling layer is implemented by transposed convolution with the kernel size of $3 \times 3$ and stride 2 . All convolutional layers have the kernel size of $3 \times 3$ and output 64 feature maps, except for the convolutional layers before the results, where each convolutional layer has the kernel size of $1 \times 1$ and outputs 3 residual maps. We pad zeros before each convolutional layer to keep the results having the same size as the input image. Moreover, the convolutional layers are followed by ReLU non-linear unit, except for the convolutional layers before the results. For the embedded Gaussian version's non-local unit, we follow the implementation of [48]. For the computational efficiency, each convolutional layer in the non-local unit has the kernel size of $1 \times 1$.

\subsection{Loss Function}

The perceptual loss has demonstrated much better visual performance than the per-pixel losses. However, it usually fails to achieve good quantitative performance [20]. To remit this issue, we incorporate the $\ell_{1}$ loss into our network optimization, which ensures the results have good quantitative scores. We only add the $\ell_{1}$ loss to stage $\mathrm{T}=3$ while the stages $\mathrm{T}=1$ and $\mathrm{T}=2$ are optimized by only using the perceptual loss because it is hard to achieve the convergence when putting these two losses on each stage's output. Besides, such an optimization scheme saves the training time.

We employ the perceptual loss based on the VGG-19 network $\phi$ [42] pre-trained on the ImageNet dataset [7]. Let $\phi_{j}(x)$ be the $j$ th convolutional layer. We measure the distance between the feature representations of the enhanced retinal image $\hat{R}$ and the ground truth image $R$ as:

$$
L_{p e r}^{3}=\sum_{i=1}^{M}\left|\phi_{j}\left(\hat{R}_{i}\right)-\phi_{j}\left(R_{i}\right)\right|,
$$

where $M$ is the number of each batch in the training procedure. Similar to the $L_{\text {per }}^{3}$ for $\mathrm{T}=3$, the losses of $\mathrm{T}=1$ and $\mathrm{T}=2$ are denoted as $L_{\text {per }}^{1}$ and $L_{\text {per }}^{2}$. The $\ell_{1}$ loss measures the difference between $\hat{R}$ and $R$ at stage 3 as:

$$
L_{\ell_{1}}^{3}=\sum_{m=1}^{H} \sum_{n=1}^{W}|\hat{R}(m, n)-R(m, n)|,
$$

where $W$ and $H$ denote the width and height of the image, rspectively.

The total loss is the linear aggregation of the multi-term loss:

$$
L_{\text {total }}=L_{\text {per }}^{1}+L_{\text {per }}^{2}+L_{\text {per }}^{3}+\lambda L_{\ell_{1}}^{3},
$$

where $\lambda$ is the weight of $L_{\ell_{1}}^{3}$ to balance different terms.

\subsection{Non-Uniform Illumination Retinal Image Synthesis}

In the real world, it is almost impossible to obtain sufficient paired retinal image with non-uniform illumination and the corresponding clear retinal image. To train the proposed NuI-Go, we approximate the non-uniform illumination degradation model of retinal images by combining the domain knowledge of retinal images with the human-lens attenuation and scattering model. With the abovementioned retinal image degradation model, we simulate large amounts of degraded retinal images for our model training.

Inspired by the human-lens attenuation and scattering model [37], the retinal image degradation can be reduced to a Retinex model [21] when only the illumination effects are considered:

$$
I_{c}(m, n)=R_{c}(m, n) \cdot L(m, n), c \in\{r, g, b\},
$$

where $I$ represents the observed retinal image; $(m, n)$ denotes the pixel coordinates; $R$ is the reflectance of the retinal image (i.e., the desired result); and $L$ is the flash illumination of the fundus camera. This simplified model can be described as that the observed retinal image $I$ is decomposed into the product of the reflectance $R$ and the illumination mask $L$. In this paper, we expect to achieve the reflectance of retinal image $R$ from the observed non-uniform 

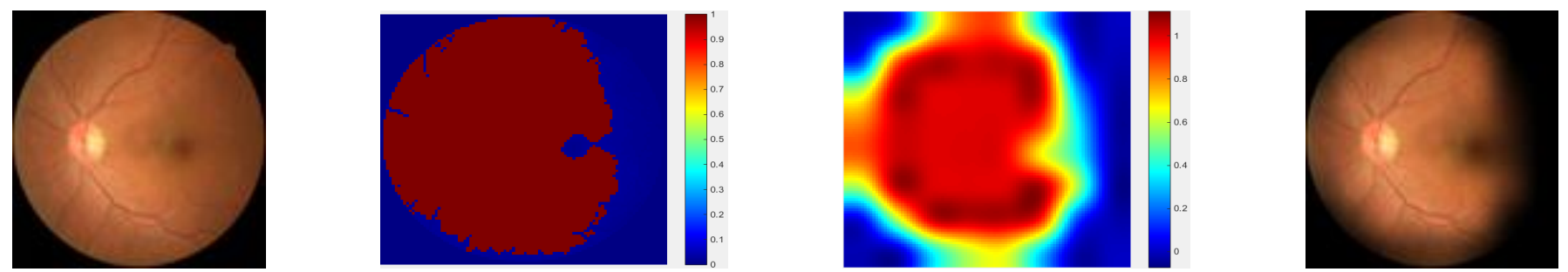

Figure 4: An example of the non-uniform illumination synthesis. From left to right are the well-lit retinal image, the coarse illumination mask, the smoothed illumination mask, and the synthetic retinal image. Different color represents different values in the illumination masks that are represented by heatmaps.

illumination retinal image $I$. Here, we assume that each channel of the retinal image has the same illumination mask. According to Eq. (7), given a clear retinal image $R$, a non-uniform illumination mask $L$ is needed to generate the non-uniform illumination retinal image $I$.

Concretely, we first transform the retinal image to the CIE Lab color space and search the well-lit regions in the illumination channel by different thresholds. If the pixel values are larger than the current threshold, they are set to 1 . Or these pixels serve as the non-uniform illumination pixels by Gamma correction with ran$\operatorname{dom} \gamma$ values ranging from 0.1 to 0.5 . The purpose is to preserve the original illumination of the well-lit regions and meanwhile make the low-light regions darker as the non-uniform illumination mask. The potential reason is that some regions in the retinal images are seldom affected by non-uniform or poor illumination, such as optic cup region. In this paper, we use five thresholds from 0.1 to 0.5 with the stride of 0.1 . We did not find much improvement when setting more thresholds. After that, a coarse non-uniform illumination mask is generated. Considering the retinal images usually have the smoothed and region-wise non-uniform illumination, we smooth the coarse non-uniform illumination mask by simple $8 \times$ down-sampling and $8 \times$ up-sampling operations. Here, other smooth approaches also can be used. Finally, a retinal image with non-uniform illumination can be synthesized by using Eq. (7).

Figure 4 presents an example of the synthetic image, where the synthetic retinal image has realistic non-uniform illumination, especially preserving the regions which are seldom affected by the non-uniform illumination.

\section{EXPERIMENTS}

\subsection{Training and Implementation Details}

We collect 2,500 well-lit retinal images from the publicly available training set of Kaggle's Diabetic Retinopathy Detection Challenge $(\mathrm{KDRDC})^{1}$ which provides retinal images taken under a variety of imaging conditions. Following the approach mentioned in Sec. 3.3, we generate 12,500 non-uniform illumination retinal images and resize them to a size of $256 \times 256$ due to our limited memory. We randomly split these synthetic images into two parts: 10,000 images for training and the rest as a testing dataset (denoted as Test A).

\footnotetext{
${ }^{1}$ https://www.kaggle.com/c/diabetic-retinopathy-//detection/
}

We implement our network with TensorFlow on a PC with an NVIDIA GTX 1080Ti GPU. During training, a batch size of 8 is applied, and the filter weights are initialized by Gaussian distribution. We use ADAM for network optimization, and fix the learning rate to $1 e^{-4}$. We compute perceptual loss at layer relu5_4 of the VGG-19 network. The weight $\lambda$ in loss function is set to 100 . The runtime of the NEDRB is 0.088 s for an image with a size of $256 \times 256$, which is fast for practical applications.

\subsection{Evaluation of Retinal Image Non-Uniform Illumination Removal}

In this section, we compare the proposed NuI-Go network with the state-of-the-art models on the synthetic and real retinal images. The synthetic retinal images are denoted as Test $\mathbf{A}$, and the real-world retinal images, denoted as Test $\mathbf{B}$, are obtained from the testing set of KDRDC, including 53,576 images taken under a variety of imaging conditions. The compared methods include recent retinal image enhancement method (SGF [4]), traditional image enhancement method (CLAHE [52]), and state-of-the-art low-light image enhancement method (LIME [14]).

The visual comparisons on synthetic and real-world retinal images are presented in Figure 5. From it, the methods of CLAHE [52] and SGF [4] have little effect on the non-uniform illumination regions. LIME [14] method improves the brightness of retinal images; however, there still remains some poor illumination regions and over-exposured regions on the results due to its limited generalization capability for fundus images. Such results may lead to a clinical misdiagnosis. In contrast, the proposed NuI-Go can effectively correction the non-uniform illumination and preserve the details and natural look of retinal images.

We further use the full-reference image quality assessment metrics PSNR and SSIM [49] for quantitative evaluations on Test A. A higher PSNR value indicates the similarity in terms of pixel-wise values, and a larger SSIM score indicates the result that is closer to the ground truth in terms of structural properties. The average quantitative scores on Test $\mathbf{A}$ are reported in Table 1. As presented in Table 1, the proposed NuI-Go achieves the best performance across all metrics. In addition, our method has overwhelming advantages than all the compared methods (CLAHE [52], LIME [14], SGF [4]) in terms of the average quantitative scores on the synthetic dataset. For example, the average PSNR value of our method is 32.2668 while the second best value (i.e., the result of LIME [14]) 


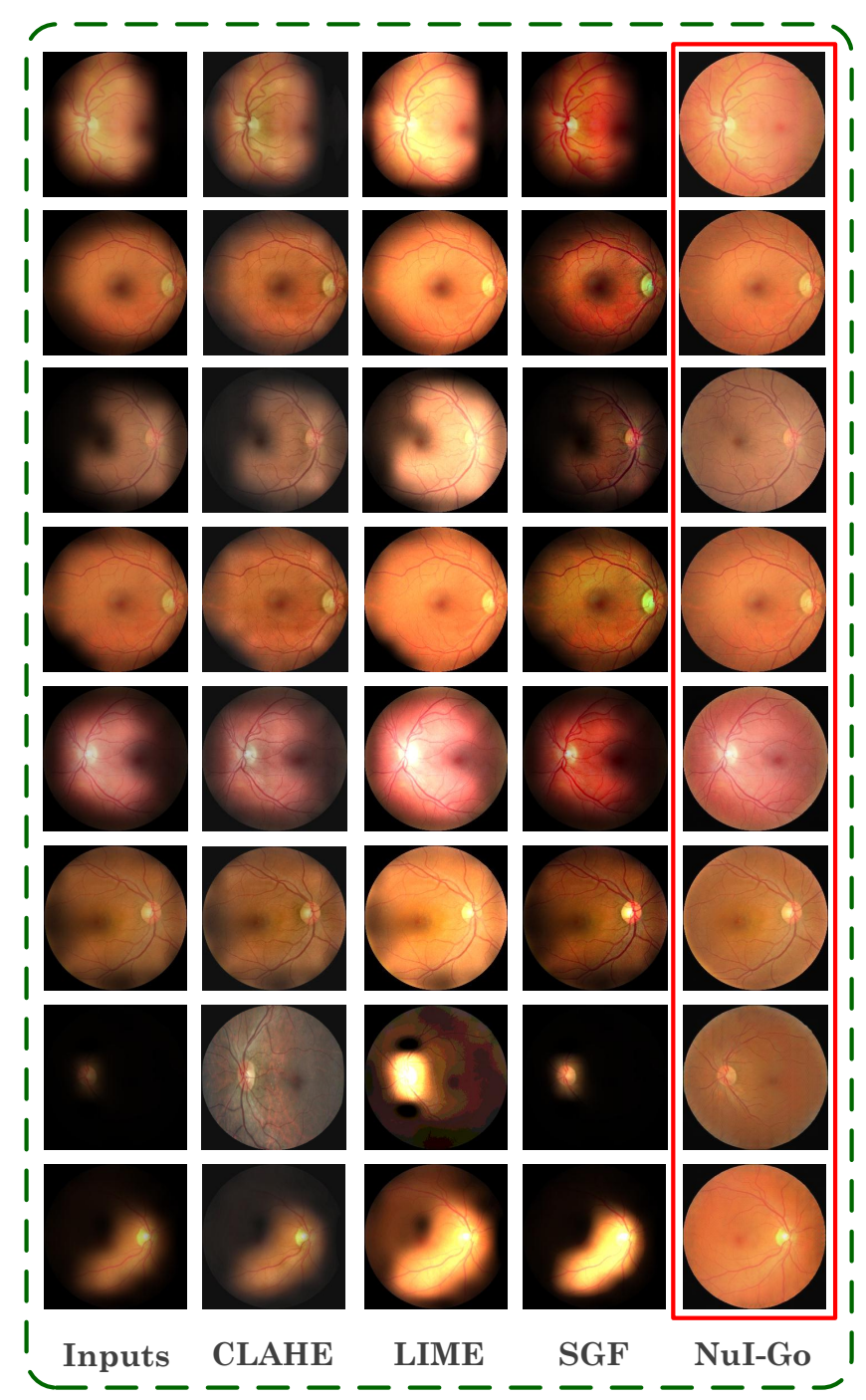

Synthetic Retinal Images from Test A

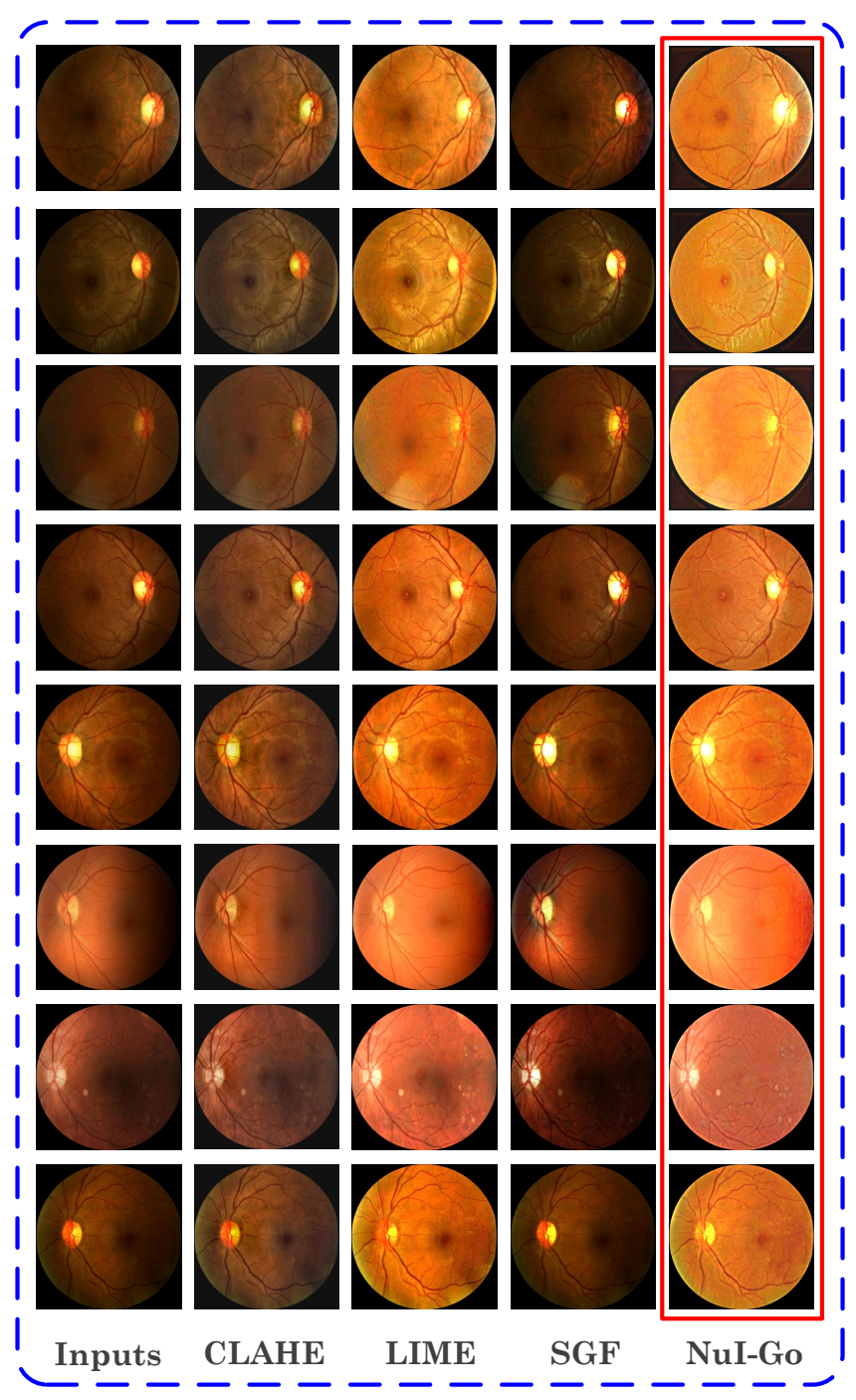

Real Retinal Images from Test B

Figure 5: Visual comparisons on synthetic and real retinal images. Red box indicates our results.

is only 16.3671 . For the average SSIM value, it has a similar tendency to that of PSNR. Such a result indicates the effectiveness of the proposed non-uniform illumination removal method.

For Test B, we employ a commonly used non-reference image quality assessment algorithm NIQE [35] to assess the perceptual quality. A smaller NIQE score indicates better perceptual quality. Besides, we also conduct a user study to provide realistic feedback about subjective quality. We first randomly select 50 images from Test B, and then invite 10 participants with image enhancement expertise to rank the results of different methods from 1 to 5 , where 1 is the worst quality and 5 corresponds to the best quality. This user study is repeated five times. The average values in terms of NIQE [35] and user study are shown in Table 2.
Table 1: Quantitative evaluations of PSNR (dB) and SSIM on Test A. For each case, the best result is in bold.

\begin{tabular}{l|l|l|l|l|l}
\hline Metrics & Inputs & CLAHE [52] & LIME [14] & SGF [4] & NuI-Go \\
\hline PSNR & 14.6994 & 16.3671 & 14.3072 & 12.2262 & $\mathbf{3 2 . 2 6 6 8}$ \\
SSIM & 0.6450 & 0.5065 & 0.6902 & 0.5558 & $\mathbf{0 . 8 2 8 1}$ \\
\hline
\end{tabular}

Table 2: The average non-reference NIQE scores and user study scores on Test B. For each case, the best result is in bold.

\begin{tabular}{l|l|l|l|l|l}
\hline Metrics & Inputs & CLAHE [52] & LIME [14] & SGF [4] & NuI-Go \\
\hline NIQE & 7.8087 & 6.9539 & 7.5331 & 8.8697 & $\mathbf{6 . 9 1 1 0}$ \\
User Study & 2.1452 & 2.5708 & 3.2416 & 2.4122 & $\mathbf{3 . 8 5 5 8}$ \\
\hline
\end{tabular}



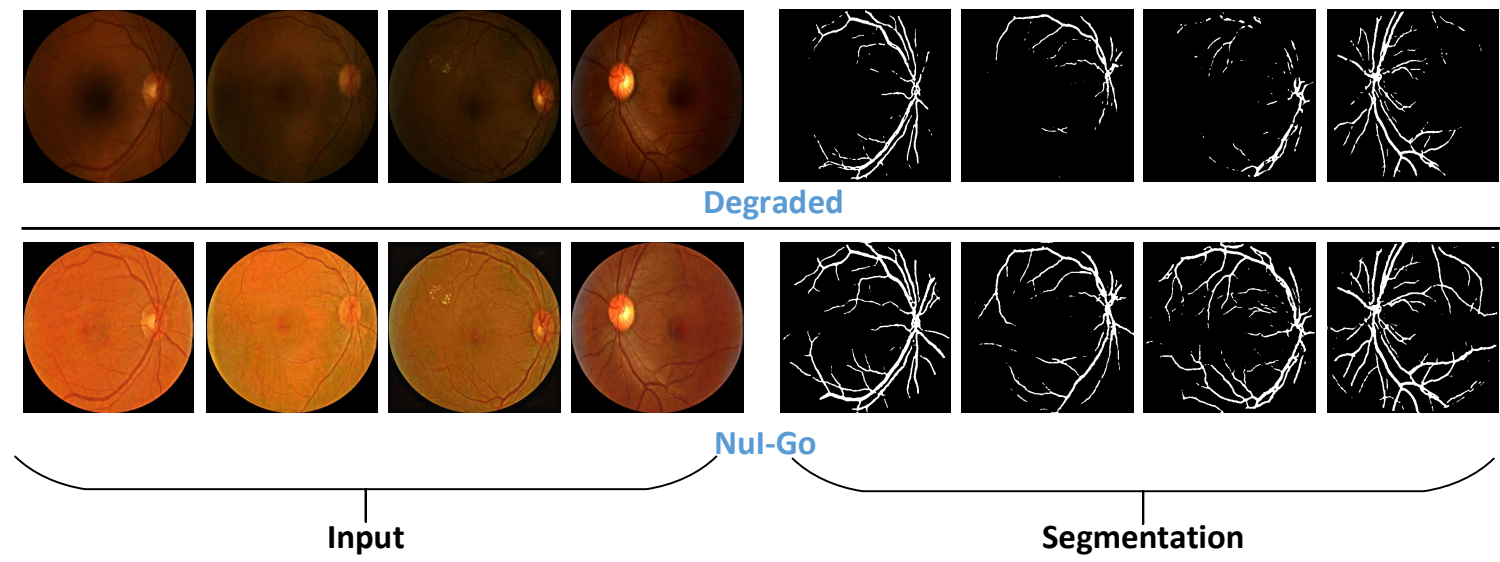

Segmentation

Figure 6: Visual enhancement and retinal vessel segmentation results. Left side: from top to bottom are the degraded retinal images via the proposed non-uniform illumination synthesis method by using the real images sampled from DRIVE dataset [43] and STARE dataset [17] and the corresponding enhanced images via the proposed NuI-Go network, respectively. Right side: from top to bottom are the segmentation results of the corresponding degraded images and the segmentation results of the enhanced images by the NuI-Go network, respectively. The segmentation results are produced by the CE-Net [11].

In Table 2, our method achieves the best performance in terms of non-reference NIQE [35] and subjective user study. Such results demonstrate our method can generalize to real retinal images and effectively improve the visual quality of degraded retinal images. Besides, it also indicates the reasonability of our non-uniform illumination synthesis approach. Though our model is trained on synthetic training data, it is also useful for real retinal images.

In addition, we found our model can be applied to other image data. It is a general model when the corresponding paired training data are available.Âă However, our network structure is more suitable for retinal image that shares structured texture and similar color. These characteristics of retinal image provide a strong prior for correcting the non-uniform illumination regions by the context.

\subsection{Extended Application of Retinal Vessel Segmentation}

To evaluate the performance of our method in practical application such as retinal vessel segmentation, we use recent retinal vessel segmentation network CE-Net [11] which is trained on DRIVE dataset [43] as a basic retinal vessel segmentation model. We found that the existing vessel segmentation datasets do not contain the non-uniform or poor illumination retinal images. However, the non-uniform illumination retinal images are ubiquitous in the real world. Therefore, we degrade the testing set of DRIVE dataset [43] and the STARE dataset [17] by the approach proposed by our nonuniform illumination synthesis algorithm. The testing data did not appear in the training set. The first manual annotation in these two datasets is used as the ground truth for performance evaluation. We enhance these testing images by different methods and feed the results to the CE-Net [11]. At last, we measure the average accuracy (Acc) and the area under receiver operation characteristic curve (AUC) by the formula mentioned in [11]. First, we present several sample results before and after processed by our method and the corresponding segmentation results in Figure 6. Then, we report the Acc and AUC scores of different methods in Table 3.
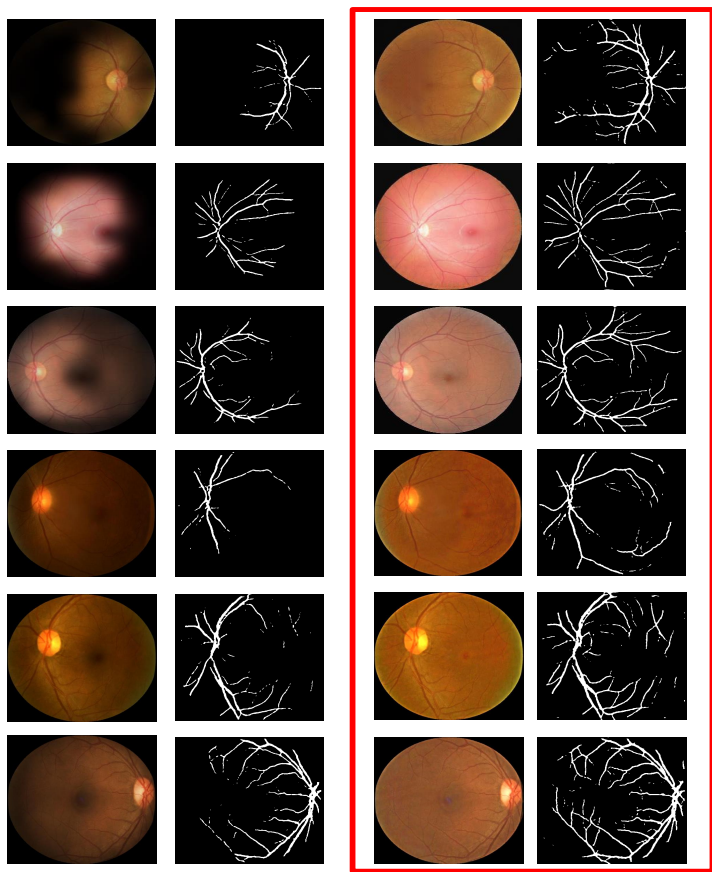

Figure 7: Visual retinal vessel segmentation results of real retinal images. Left side: the input images from Test $B$ and the corresponding segmentation results. Right side: our enhanced results and the corresponding segmentation results. The segmentation results are produced by the CE-Net [11]. Red box indicates our results. 
Table 3: Quantitative evaluations on DRIVE dataset/STARE dataset in terms of Acc and AUC. For each case, the best result is in bold.

\begin{tabular}{l|l|l|l|l|l}
\hline Metrics & Degraded Inputs & CLAHE [52] & LIME [14] & SGF [4] & NuI-Go \\
\hline Acc & $0.9508 / 0.9369$ & $0.9497 / 0.9331$ & $0.9518 / 0.9384$ & $0.9382 / 0.9322$ & $\mathbf{0 . 9 5 4 3 / 0 . 9 4 1 7}$ \\
AUC & $0.9329 / 0.7976$ & $0.9570 / 0.8509$ & $0.9746 / 0.9076$ & $0.9074 / 0.8014$ & $\mathbf{0 . 9 7 5 6 / 0 . 9 2 1 3}$ \\
\hline
\end{tabular}

In Figure 6, our method can effectively unveil the vessel under dark regions. As a result, the vessel of retinal images processed by our method can be easily segmented by the CE-Net [11] which is trained by using well-lit retinal images. Thus, despite the retinal image segmentation algorithms did not consider the non-uniform illumination issue in their designs and training procedures, our method could be used as an effective pre-processing step for further improving their performance. As presented in Table 3, the results enhanced by the proposed NuI-Go achieve better segmentation performance on the DRIVE [43] and STARE [17] datasets. Compared with the degraded image inputs, the Acc increases from 0.9508/0.9369 to $0.9543 / 0.9417$, and the AUC increases from $0.9329 / 0.7976$ to $0.9756 / 0.9213$, respectively. This fully illustrates the positive effect of the proposed NuI-Go enhancement method on retinal vessel segmentation task. In addition, it is interesting that some enhancement algorithms decrease the performance of retinal vessel segmentation, such as SGF method. The unstable results are undesired in practical diagnose applications.

To further demonstrate the robustness of our method on real retinal images, we provide several sample segmentation results for real retinal images in Figure 7. It is obvious that after enhancing by our method, some retinal vessels hidden in dark are recovered and thus can be accurately segmented by the retinal vessel segmentation network CE-Net [11]. The results in Figure 7 further demonstrate the effectiveness and robustness of our method, which is significant for practical applications.

\section{CONCLUSION}

In this paper, we propose a deep learning-based retinal image nonuniform illumination removal method. The non-uniform illumination is progressively removed via recursive residual learning while the details and color of retinal images are recovered by the combination of local and non-local features. In addition, to drive the training of the proposed NuI-Go network, we propose a non-uniform illumination degradation model of retinal image by combining the domain knowledge of retinal images with the human-lens attenuation and scattering model. This model can simulate amounts of degraded retinal images with non-uniform illumination and also can be used as a guide for full-reference image quality assessment, which calls for the development of retinal image enhancement. Experiments on synthetic and real-world retinal images show that the proposed method outperforms existing image enhancement methods. Additionally, application experiments suggest that our method can effectively improve retinal vessel segmentation, which is important for practical diagnose application.

\section{ACKNOWLEDGMENTS}

This work was supported by the National Key Research and Development Program of China (Grant No. 2017YFC0820601), the National Natural Science Foundation of China (Grant No. 61720106004), the Fundamental Research Funds for the Central Universities (Grant No. 2019RC039), and the China Postdoctoral Science Foundation (Grant No. 2019M660438).

\section{REFERENCES}

[1] M. D. Abramoff, M. K. Garvin, and M. Sonka. 2010. Retinal imaging and image analysis. IEEE Rev. Biomed. Eng. 3 (2010), 169-208.

[2] T. Celik and T. Tjahjadi. 2001. Contextual and variational contrast enhancememt. IEEE Trans. Image Process. 20, 12 (2001), 3431-3441.

[3] W. Chen, W. Wang, W. Yang, and J. Liu. 2018. Deep retinex decomposition for low-light enhancement. In Proc. of British Mach. Vis. Conf. (BMVC). 1-12.

[4] J. Cheng, Z. Li, Z. Gu, H. Fu, D. Wong, and J. Liu. 2018. Structure-preserving guided retinal image filtering and its application for optic disc analysis. IEEE Trans. Med. Imag. 37, 11 (2018), 2536-2546.

[5] S. Chuaudhuri, S. Chatterjee, N. Katz, M. Nelson, M. Goldbaum, and J. Liu. 1989. Detection of blood vessels in retinal images using two-dimensional matched filters. IEEE Trans. Med. Imag. 8, 3 (1989), 263-269.

[6] D. Coltuc, P. Bolon, and J. Chassery. 2006. Exact histogram specification. IEEE Trans. Image Process. 15, 5 (2006), 1143-1152.

[7] J. Deng, W. Dong, R. Socher, L. Li, and L. Fei-Fei. 2009. Imagenet: A large-scale hierarchical image database. In Proc. of IEEE Int. Conf. Comput. Vis. Pattern Rec. (CVPR). 248-255.

[8] P. Feng, Y. Pan, B. Wei, W. Jin, and D. Mi. 2007. Enhacing retinal image by the Contourlet transform. Pattern Rec. Lett. 28, 4 (2007), 516-522.

[9] H. Fu, J. Cheng, Y. Xu, D. Wong, J. Liu, and X. Cao. 2018. Joint optic disc and cup segmentation based on multi-label deep network and polar transformation. IEEE Trans. Med. Imag. 37, 7 (2018), 1597-1605.

[10] X. Fu, D. Zeng, Y. Huang, X. Zhang, and X. Ding. 2016. A weighted variational model for simultaneous reflectance and illumination estimation. In Proc. of IEEE Int. Conf. Comput. Vis. Pattern Rec. (CVPR). 2782-2790.

[11] Z. Gu, J. Cheng, H. Fu, K. Zhou, H. Hao, Y. Zhao, T. Zhang, S. Gao, and J. Liu. 2019. CE-Net: Context Encoder Network for 2D Medical Image Segmentation. IEEE Trans. Med. Imag. 38, 10 (2019), 2281-2292.

[12] C. Guo, C. Li, J. Guo, R. Cong, H. Fu, and P. Han. 2018. Hierarchical features driven residual learning for depth map super-resolution. IEEE Trans. Image Process. 28, 5 (2018), 2545-2557.

[13] C. Guo, C. Li, J. Guo, C. C. Loy, J. Hou, S. Kwong, and R. Cong. 2020. Zeroreference deep curve estimation for low-light image enhancement. In Proc. of IEEE Int. Conf. Comput. Vis. Pattern Rec. (CVPR). 1780-1789.

[14] X. Guo, Y. Li, and H. Ling. 2017. LIME: Low-light image enhancement via illumination map estimation. IEEE Trans. Image Process. 26, 2 (2017), 982-993.

[15] A. F. M. Hani and H. A. Nugroho. 2009. Retinal vasculature enhancement using independent component analysis. f. Biomed. Sci. Eng. 2, 7 (2009), 543-549.

[16] K. He, X. Zhang, S. Ren, and J. Sun. 2016. Deep residual learning for image recognition. In Proc. of IEEE Int. Conf. Comput. Vis. Pattern Rec. (CVPR). 770-778.

[17] A. Hoover, V. Kouznetsova, and M. Goldbaum. 1998. Locating blood vessels in retinal images by piece-wise threhshold probing of a matched filter resonse. In Proc. American Med. Inform. Asso. Sympo. 931-935.

[18] H. Ibrahim and N. Kong. 2007. Brightness preserving dynamic histogram equalization for image contrast enhancement. IEEE Trans. Consum. Electron. 53, 4 (2007), 1752-1758.

[19] P. Jiang, X. Wang, Q. Li, L. Jin, and S. Li. 2019. Correlation-aware sparse and low-rank constrained multi-task learning for longitudinal analysis of alzheimer's disease. IEEE 7. Biomed. Health Inform. 23, 4 (2019), 1450-1456.

[20] J. Johnson, A. Alahi, and L. Fei-Fei. 2016. Perceptual losses for real-time style transfer and super-resolution. In Proc. of Eur. Conf. Comput. Vis. (ECCV). 694-711.

[21] E. Land. 1986. An alternative technique for the computation of the designator in the Retinex theory of color vision. Nati. Acad. Sci. 83, 10 (1986), 3078-3080. 
[22] C. Lee, C. Lee, and C. Kim. 2013. Contrast enhancement based on layered difference representation of $2 \mathrm{~d}$ histograms. IEEE Trans. Image Process. 22, 12 (2013), 5372-5384.

[23] E. Lee. 1977. The retinex theory of color vision. Scientific American 237, 6 (1977), $108-128$.

[24] C. Li, S. Anwar, and F. Porikli. 2020. Underwater scene prior inspired deep underwater image and video enhancement. Pattern Rec. 98 (2020), 1-11.

[25] C. Li, R. Cong, J. Hou, S. Zhang, Y. Qian, and S. Kwong. 2019. Nested network with two-stream pyramid for salient object detection in optical remote sensing images. IEEE Trans. Geosci. Remote Sens. 57, 11 (2019), 9156-9166.

[26] C. Li, J. Guo, R. Cong, and J. Gong. 2017. A hybrid method for underwater image correction. Pattern Rec. Lett. 94 (2017), 62-67.

[27] C. Li, J. Guo, R. Cong, Y. Pang, and B. Wang. 2016. Underwater image Enhancement by dehazing with minimum information loss and histogram distribution prior. IEEE Trans. Image Process. 25, 12 (2016), 5664-5677.

[28] C. Li, J. Guo, and C. Guo. 2018. Emerging from water: Underwater image color correction based on weakly supervised color transfer. IEEE Signal Process. Lett 25, 3 (2018), 323-327.

[29] C. Li, J. Guo, F. Porikli, and Y. Pang. 2018. LightenNet: A convolutional neural network for weakly illuminated image enhancement. Pattern Rec. Lett. 104 (2018), $15-22$.

[30] H. Li and O. Chutatape. 2004. Automated feature extraction in color retinal images by a model based approach. IEEE Trans. Biomed. Eng. 51, 2 (2004), 246-254.

[31] M. Li, J. Liu, W. Yang, X. Sun, and Z. Guo. 2018. Structure-revealing low-light image enhancement via robust retinex model. IEEE Trans. Image Process. 27, 6 (2018), 2828-2841

[32] Y. Li and M. Brown. 2014. Single image layer separation using relative smoothness In Proc. of IEEE Int. Conf. Comput. Vis. Pattern Rec. (CVPR). 2752-2759.

[33] K. Lore, A. Akintayo, and S. Sarkar. 2017. LLNet: A deep autoencoder approach to natural low-light image enhancement. Pattern Rec. 61 (2017), 650-662.

[34] A. Mitra, S. Roy, S. Soy, and S. Setua. 2018. Enhancement and restoration of non-uniform illuminated fundus image of retina obtained through thin layer of cataract. Comput. Methods Programs in Biomedicine 156 (2018), 169-178.

[35] A. Mittal and R. Soundararajan. 2013. Making a completely blind image quality analyzer. IEEE Signal Process. Lett. 20, 3 (2013), 209-212.

[36] A. Moreau, P. Anderer, M. Ross, A. Cerny, T. Almazan, and B. Peterson. 2018. Detection of nocturnal scratching movements in patients with atopic dermatitis using accelerometers and recurrent neural networks. IEEE 7. Biomed. Health Inform. 22, 4 (2018), 1011-1018.

[37] E. Peli and T. Peli. 1989. Restoration of retinal images obtained through cataracts. IEEE Trans. Med. Imag. 8, 4 (1989), 401-406.

[38] W. Ren, S. Liu, L. Ma, Q. Xu, X. Xu, X. Cao, J. Du, and M. Yang. 2019. Low-light image enhancement via a deep hybrid network. IEEE Trans. Image Process. 28, 9 (2019), 4364-4375.

[39] C. Rother, M. Kiefei, L. Zhang, B. Scholkopf, and P. Gehler. 2011. Recovering intrinsic images with a global sparsity prior on reflectance. In Adcances in Neural Inf. Process. Sys. (NeuralPS). 765-773.

[40] S. Saha, A. Fletcher, D. Xiao, and Y. Kanagasingam. 2018. A novel method for authomated correction of non-uniform/poor illumination of retinal images without creating false artifacts. F. Vis. Commun. Image Rep. 51 (2018), 95-103.

[41] W. Shao, Z. Han, J. Cheng, L. Cheng, T. Wang, L. Sun, Z. Lu, J. Zhang, D. Zhang, and K. Huang. 2020. Integrative analysis of pathological images and multidimensional genomic data for early-stage cancer prognosis. IEEE Trans. Med. Imag. 39, 1 (2020), 99-110.

[42] K. Simonyan and A. Zisserman. 2014. Very deep convolutional networks for large-scale image recognition. arXiv preprint arXiv: 1409.1556 (2014).

[43] J. Staal, M. Abramoff, M. Niemeijer, M. Viergever, and B. Ginneken. 2004. Ridgebased vessel segmentation in color images of the retina. IEEE Trans. Med. Imag. 23, 4 (2004), 501-509.

[44] J. Stark. 2000. Adaptive image contrast enhancement using generalizations of histogram equalization. IEEE Trans. Image Process. 9, 5 (2000), 889-896.

[45] E. Swanson, J. Izatt, M. Hee, D. Huang, C. Lin, J. Schuman, C. Puliafito, and J. Fujimoto. 1993. In vivo retinal imaging by optical coherence tomography. Optic. Lett. 18, 21 (1993), 1864-1866.

[46] R. Wang, Q. Zhang, C. Fu, X. Shen, W. Zheng, and J. Jia. 2019. Underexposed phto enhancement using deep illumination estimation. In Proc. of IEEE Int. Conf. Comput. Vis. Pattern Rec. (CVPR). 6849-6857.

[47] S. Wang, J. Zheng, H. Hu, and B. Li. 2013. Naturalness preserved enhancement algorithm for non-uniform illumination images. IEEE Trans. Image Process. 22, 9 (2013), 3538-3548.

[48] X. Wang, R. Girshick, A. Gupta, and K. He. 2018. Non-local neural networks. In Proc. of IEEE Int. Conf. Comput. Vis. Pattern Rec. (CVPR). 7794-7803.

[49] Z. Wang, A. Bovik, H. Sherikh, and E. Simoncelli. 2004. Image quality assessment: From error visibility to structural similarity. IEEE Trans. Image Process. 13, 8 (2004), 600-612.

[50] Z. Yan, X. Yang, and K. Cheng. 2019. A three-stage deep learning model for accurate retinal vessel segmentation. IEEE f. Biomed. Health Inform. 23, 4 (2019), $1427-1435$.
[51] M. Zhou, K. Jin, S. Wang, J. Ye, and D. Qiao. 2018. Color retinal image enhancement based on luminosity and contrast adjustment. IEEE Trans. Biomed. Eng. 65, 3 (2018), 521-527.

[52] Karel Zuiderveld. 1994. Contrast Limited Adaptive Histogram Equalization. 